\title{
Treatment gaps of epilepsy and retention rates of sodium valproate in rural Guangxi, China
}

\author{
D.H. Huang, J.O. Zheng, J. Chen and L. Yu \\ Department of Neurology, The First Affiliated Hospital, \\ Guangxi Medical University, Nanning, Guangxi, China \\ Corresponding author: J.O. Zheng \\ E-mail: dhjzcn@yeah.net
}

Genet. Mol. Res. 13 (3): 6202-6212 (2014)

Received May 24, 2013

Accepted October 22, 2013

Published August 15, 2014

DOI http://dx.doi.org/10.4238/2014.August.15.3

\begin{abstract}
This study aimed to investigate the prevalence and treatment gaps of epilepsy, as well as the clinical effects, drug safety, and retention rates of sodium valproate (VPA) for treating epilepsy. Physicians received supervised training to use the survey form recommended by the Chinese Association Against Epilepsy while screening for suspected or confirmed epilepsy cases. These cases were ultimately enrolled in the study by neurologists. Enrolled patients were given a year of free VPA treatment so that its efficacy and adverse effects during the follow-up period could be evaluated. In total, 302 patients were enrolled in this study, which included 189 males and 113 females. Among these cases, 179 (59.27\%) were confirmed to have generalized seizures, $162(53.6 \%)$ had tonic-clonic seizures, $10(3.3 \%)$ had absence seizures, and $123(40.72 \%)$ had partial seizures. Only 63 patients had received regular treatments 1 week before enrollment, with a treatment gap of $79.1 \%$. The retention rates during the 6th, 12th, 18th, and 24th months were 100, 93.56, 89.05 , and $77.06 \%$, respectively. During the 1-year follow-up period, 30 cases encountered mild adverse effects, but no severe adverse reactions were reported. A large treatment gap for epilepsy still existed in the rural areas of southern China, with few adverse effects and high
\end{abstract}


retention rates. VPA showed satisfactory effects in the treatment of epileptic patients.

Key words: Epilepsy; Sodium valproate; Retention rates

\section{INTRODUCTION}

Epilepsy is a common disease of the central nervous system that affects 50 million patients around the world, among which $80 \%$ live in resource-poor developing countries (Leonardi and Ustun, 2002). Approximately $90 \%$ of patients with epilepsy in these countries do not have access to appropriate treatments (Scott et al., 2001). The prevalence of epilepsy in China is 6-7\% with approximately 9 million patients nationwide, 5-6 million of which have active epilepsy, and an annual increase of 400,000 new cases has been reported (Wu, 2006). The main treatment for epilepsy is the use of antiepileptic drugs. However, treatment gaps exist, especially in the rural areas of China. The lack of medical knowledge, poor economic backgrounds, and inappropriate transport conditions hinder approximately twothirds of patients with epilepsy from accepting or receiving formal treatments (Wang et al., 2002b). The Chinese Ministry of Health and the Mental Health Division of the World Health Organization (WHO) launched the "Epilepsy Demonstration Project" under the leadership and support of the Disease Control Division in 2000. This project included 92 villages and towns in 6 domestic provinces of 8 counties. After the screening, treatment, and follow-up management of convulsive epileptic patients using phenobarbital, the study concluded that phenobarbital was a convenient, inexpensive, and effective drug for epilepsy, with few side effects. However, phenobarbital belongs to the second category of psychiatric medication, and its prescription is under stringent control. Thus, doctors can only administer this drug in weekly doses, which makes it unsuitable for rural patients. By contrast, sodium valproate (VPA) is a broad-spectrum antiepileptic drug that has obvious curative effects on simple partial seizures, as well as complex partial, myoclonic, atonic, absence, and generalized tonic-clonic seizures (Wang et al., 2011). A seminar on the treatment of adult epilepsy by VPA that was held in Goteborg in June of 2005 revealed that VPA was considered as an effective drug for the treatment of various types of epilepsy. Thus, VPA should be considered as a first-line treatment of juvenile myoclonic epilepsy as well as a suitable form of monotherapy for other congenital forms of generalized epilepsy (Ben-Menachem et al., 2006). Gekht (2012) evaluated the use of VPA (depakine chrono) in adult patients with partial epilepsy in a multi-center prospective non-comparative study, and the results showed high efficacy and good tolerability of depakine chrono as first-line monotherapy in adult patients with partial epilepsy. To date, several studies have investigated the curative effects of VPA. A survey showed that a combination of VPA and levetiracetam was effective in all forms of epilepsy. There were positive changes in the frequency of seizures and epileptic activity based on electroencephalography results (Karlov et al., 2012). However, none of these studies provided detailed information or analyses of the clinical efficacy, side effects, or follow-up of VPA using a large sample of epileptic patients in rural areas. Wang et al. (2011) evaluated the curative effects of VPA in 607 epileptic patients in the rural areas of China and found that VPA was similar to phenobarbital as a more effective drug for epilepsy with less serious adverse reactions. VPA is a broad-spectrum anticonvulsant whose effects and side effects 
can be easily controlled by primary care physicians; thus, it can be recommended for use in rural areas of China (Wang et al., 2011). The "Chinese Rural Areas for Epilepsy Prevention and Management Demonstration Project" using phenobarbital showed the drug's high antiepileptic efficacy with less adverse reactions. Wang et al. (2012) also studied the effects of VPA on epilepsy in 532 patients in rural China. In the 12-month assessment, VPA showed favorable efficacy, few side effects, and overall good acceptability. It was also relatively cheap. Therefore, VPA was suggested to be a suitable alternative to phenobarbital for treating convulsive epilepsy in rural areas of China (Wang et al., 2012). Here, we report results of an investigation conducted in cooperation with the Chinese Anti-Epilepsy Association on the curative effects of VPA in the rural areas of Guangxi Province based on the model of the "Chinese Rural Areas Epilepsy (Phenobarbital) Treatment Project Management". Understanding the current situation with respect to the incidence rate, treatment gap, and drug therapy of epilepsy, as well as the VPA efficacy and its influencing factors, in the rural areas of Guangxi is important. Such information could contribute to the future administration of drug treatments as well as the formulation of future alternative drugs for epilepsy in the rural areas of China.

\section{MATERIAL AND METHODS}

\section{Patients}

All confirmed or suspected cases of epilepsy from March 2007 to May 2008 were investigated. The epileptic patients were aged from 6 to 70 years old. The patients were from 4 counties of Guangxi, namely, Tiandong, Tianyang, Tiandeng, and Pingguo. This study was conducted in accordance with the declaration of Helsinki and with approval from the Ethics Committee of the First Affiliated Hospital, Guangxi Medical University. Written informed consent was obtained from all participants.

\section{Criteria}

First, physicians from the above-mentioned counties and villages underwent training before they were given the screening questionnaire that was issued by the Chinese AntiEpileptic Association. These questionnaires were used to screen for confirmed or suspected cases of epilepsy. Neurologists then individually interviewed each patient using the "Review of Neurology Enrollment Scale". The subjects that fit the criteria and had a confirmed diagnosis of epilepsy were enrolled in this study. The following inclusion criteria were used for the selection of patients: 1) patients were diagnosed based on the criteria for the classification of epilepsy and epileptic syndromes of the 1989 International Epilepsy Alliance, 2) patients should have experienced at least two episodes of seizure within the past year, 3) patients or their guardians signed the informed consent forms and approved the use of VPA treatment during the survey.

The exclusion criteria were as follows: 1) breastfeeding or pregnant women, 2) patients with serious heart, lung, liver, kidney, and other organ diseases, 3) a history of mental illness or the use of corresponding medication, 4) a history of addiction or abuse of alcohol or drugs. 


\section{Medication}

The total starting dosage of the medication for adults was $600 \mathrm{mg}$ /day for the first week, which was divided into $200 \mathrm{mg}$ three times a day after meals. The medication dose was maintained in the absence of seizures. However, the dose was increased by $200 \mathrm{mg}$ per week if seizures occurred, for as long as no adverse reactions were documented and until episodes were sufficiently controlled; the daily doses should not exceed $1600 \mathrm{mg}$. The total initial dosage for children was $20 \mathrm{mg} \cdot \mathrm{kg}^{-1} \cdot \mathrm{day}^{-1}$, which was divided into 2 doses after meals. The medication dose was maintained in the absence of seizures. If there were still attacks, the dose was increased to $5-10 \mathrm{mg} / \mathrm{kg}$ per week, for as long as no adverse reactions were documented and until episodes were sufficiently controlled; the daily doses should not exceed $30 \mathrm{mg} \cdot \mathrm{kg}^{-1} \cdot$ day $^{-1}$.

\section{Efficacy criteria}

The efficacy of treatment was determined in three categories: obvious improvement, which was defined as 6 months without attacks or if the frequency of attacks reduced by more than $75 \%$; effective improvement, which was defined if the frequency of attacks reduced by 50 to $75 \%$; and no improvement, which was defined as no reduction in frequency of seizures, a reduction in the frequency of attacks below $50 \%$, or increased attack frequency over $25 \%$. The retention rate of treatment was defined as the proportion of patients who were still using the drug after $6,12,18$, and 24 months from the time of enrollment in the study and acceptance of treatment.

\section{Statistical analysis}

The follow-up data of all patients enrolled in the study were recorded using the EpiData (version 3.0) software. The SPSS (version 13) software was used for statistical analysis. Rate comparisons were assessed using the $\chi^{2}$ test and statistically significant differences were defined at $\mathrm{P}<0.05$.

\section{RESULTS}

\section{General information}

In total, 302 patients were treated using VPA, of which 189 were men and 113 were women. Among these patients, the average age was 29.2 years, with 223 cases within the range of 10 to 39 years old (73.8\%), and 91 cases within the range of 20 to 29 years old (30.1\%). The average age of the onset of attacks was 20.3 years old, and 235 cases $(77.8 \%)$ started at the age of 29 years. Systemic attacks occurred in 179 cases $(59.27 \%)$, including 10 cases $(3.3 \%)$ of absence seizures and 162 cases $(53.6 \%)$ of tonic-clonic seizures. Partial seizures occurred in 123 cases $(40.7 \%)$, including 95 cases $(31.5 \%)$ of secondary body seizures and 14 cases $(4.6 \%)$ of simple and complex partial seizures. Idiopathic epilepsy occurred in 214 cases (70.9\%), including 88 cases $(29.1 \%)$ of symptomatic epilepsy. Among the patients with symptomatic epilepsy, 28.4, 21.6, 20.5, and $15.9 \%$ of the cases had ebrile convulsions, traumatic brain injury, intracranial infections, and cerebral cysticercosis as the main causes of epilepsy. 


\section{Treatment gap}

Of the 302 surveyed patients with active epilepsy, 154 patients had a history of antiepileptic drug therapy, 63 had regular treatment a week before enrollment, 48 had irregular treatment, and 191 were untreated. These results indicated an overall treatment gap of $79.1 \%$.

\section{Retention rates}

The retention rate was defined as the proportion of withdrawal in non-cured patients from the start of the investigation to the end.

During the first 6 months, all 302 patients were followed-up. Among these patients, 7 cases withdrew from the study, including 2 cases that were lost, 4 cases that withdrew because of no effective results or deterioration of symptoms, and 1 case that retreated because of side effects. Thus, 295 cases were still under medication after 6 months, with a retention rate of $97.68 \%$.

Between 6 and 12 months, 19 patients were cured and decided to withdraw. Thus, 276 cases were still under medication after 12 months, with a retention rate of $97.68 \%$.

Between 12 and 18 months, 25 cases were withdrawn, including 12 that were lost and 13 that were cured. Thus, a total of 251 cases were still under medication after 18 months, with a retention rate of $93.33 \%$.

Between 18 and 24 months, 154 cases were withdrawn, including 26 that were lost, 27 that withdrew because they were cured, and 127 cases that retreated because of no obvious improvement or other reasons. Thus, 97 cases were still under medication by the end of the study, with a retention rate of $43.42 \%$.

The probability of reserves represented the proportion of non-cured patients that withdrew during the 2-year observation period during the different 6-month intervals.

After 6, 12, 18, and 24 months, the probabilities of reserves were 97.68, 100.0, 95.5, and $46.53 \%$, respectively (Table 1 and Figure 1 ).

\begin{tabular}{|c|c|c|c|c|c|c|c|}
\hline $\begin{array}{l}\text { Follow-up after } \\
\text { treatment (months) }\end{array}$ & $\begin{array}{l}\text { Total enrolled } \\
\text { cases }\left(N^{o}\right)\end{array}$ & $\begin{array}{l}\text { Withdrawn cases - } \\
\text { cured }\end{array}$ & $\begin{array}{l}\text { Number of cases - } \\
\text { preliminary }\end{array}$ & $\begin{array}{l}\text { Number of cases - } \\
\text { corrected }\end{array}$ & $\begin{array}{c}\text { Non-cured and } \\
\text { withdrawal-medication (\%) }\end{array}$ & $\begin{array}{c}\text { Reserves } \\
(\%)\end{array}$ & $\begin{array}{l}\text { Retentions } \\
(\%)\end{array}$ \\
\hline 0 to 6 & 7 & 0 & 302 & 298.5 & 2.32 & 97.68 & 97.68 \\
\hline 6 to 12 & 0 & 19 & 295 & 295 & 0 & 100.00 & 97.68 \\
\hline 12 to 18 & 12 & 13 & 276 & 270 & 4.5 & 95.50 & 93.33 \\
\hline 18 to 24 & 127 & 27 & 251 & 200.5 & 53.47 & 46.53 & 43.42 \\
\hline
\end{tabular}

P.B. $=$ Drug treatment of patients after more than 24 months for a total of 97 cases.

\section{Adverse reactions}

During the first year of follow-up, 30 cases with adverse reactions were documented, with an incidence of $9.93 \%$. Among these cases, 6 (20.00\%) were fatigue, $4(13.33 \%)$ were lethargy, $3(30.00 \%)$ were memory loss, abnormal liver functions, and dizziness, 2 cases $(26.67 \%)$ were weight gain, overeating disorder, headache, and tremors, and 1 case (10\%) was hair loss, unsteady gait, and loss of appetite. 


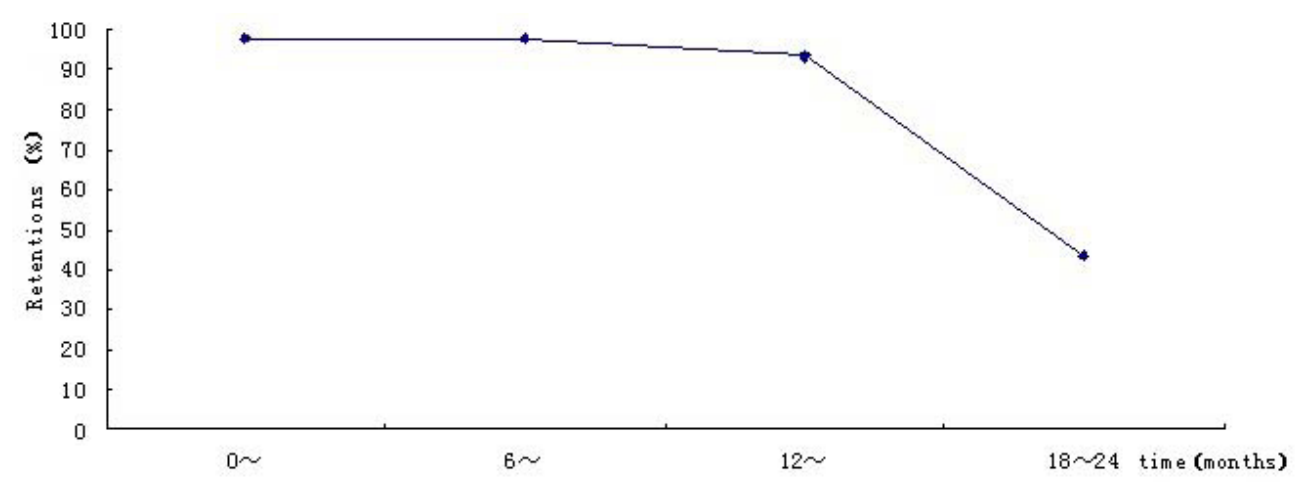

Figure 1. Probability of treatment by sodium valproate.

\section{DISCUSSION}

The WHO report showed that developed, transitioning, developing, and underdeveloped countries had prevalence rates of epilepsy of 5.0, 6.1, 7.2, and 11.2\%, respectively (Jallon, 1997). The present study examined the prevalence status and treatment gaps of epilepsy, as well as the use of VPA, in the treatment of patients in some rural areas in Guangxi Province. After 2 years of follow-up, the efficacy, retention rates, and occurrence of adverse reactions during VPA treatment provided strong evidence of the potential of VPA for antiepileptic therapy in rural areas.

This study showed that the age distribution of epileptic patients was in the range of 10 to 39 years. Most patients belonged to the 20- to 29-year-old age group, which formed the first peak of the onset of epilepsy. These findings were consistent with results of a survey conducted in the rural area of Heilongjiang Province (Wang et al., 2002a). The observed seizure attacks in this study are also consistent with national findings. Generalized seizures are the most common forms, particularly the generalized tonic-clonic seizures. Unlike in the United States, partial seizures are the most common form in the United Kingdom and other developed countries, which account for as much as over 50\% of all reported seizures (Sridharan and Murthy, 1999). This difference may be attributed to the fact that these countries prioritize generalized tonic-clonic seizures. Thus, other forms of seizure cannot be ruled out in other regions.

The WHO/International Bureau for Epilepsy/International League Against Epilepsy reported that the treatment gap of epilepsy in developing countries was between 60 and $98 \%$. According to the WHO, the prevalence of epilepsy is the highest in low- and lower middle-income countries, which includes over $80 \%$ of the countries of sub-Saharan Africa, where the majority of people with epilepsy are not receiving appropriate care. In sub-Saharan Africa, shortages of trained health workers, limited diagnostic equipment, inadequate antiepileptic drug supplies, cultural beliefs, and social stigma contribute to the large treatment gap for epilepsy (Chin, 2012).

\section{Global disparities in the epilepsy treatment gap}

A systematic review showed that the treatment gap was over $75 \%$ in low-income countries and was over 50\% in most lower middle- and upper middle-income countries, whereas 
many high-income countries had gaps of less than $10 \%$ (Meyer et al., 2010). A previous study (Mbuba et al., 2008) reported that the treatment gap could reach above 56\% in poor, developing countries, of which 55\% are in Latin America, 64\% in Asia, and 49\% in Africa, with $73 \%$ in their rural areas and $47 \%$ in their cities. This study showed that these treatment gaps were due to treatment costs, which accounted for $62 \%$ (11 to $90 \%$ ), no effective amelioration of symptoms despite antiepileptic drug use, which accounted for 53\% (18 to 44\%), belief in traditional treatment methods, which accounted for $44 \%$ (6 to $82 \%$ ), and superstition or other cultural beliefs, which accounted for $40 \%$ ( 7 to $65 \%$ ). The treatment gap for people living with epilepsy in Madagascar was reported to be $92 \%$ (Ratsimbazafy et al., 2011). Similarly, China's rural areas were reported to have treatment gaps of $97.1 \%$ in rural areas of Tibet (Zhao et al., 2007), $70.73 \%$ in Shanghai rural areas (Ding et al., 2004), and 62.6\% in five rural provinces of China. In the present study, a treatment gap of $79.1 \%$ was observed, which is similar to those of other rural areas in China. Wang et al. (2003) surveyed 55,000 people and found that the epilepsy prevalence rate was $7 \%$. Among the patients in their study, $41 \%$ had not received proper treatments, $4.6 \%$ had active epilepsy, and $63 \%$ did not receive any antiepileptic drug treatment during the week before the survey (Wang et al., 2003). The treatment gap of epilepsy differs greatly in different countries and in different regions. This difference is greater than $75 \%$ in low-income countries, greater than $50 \%$ in middle- to high-income countries, and less than 10\% in high-income countries (Meyer et al., 2010). Meinardi et al. (2001) confirmed that the quality of health care facilities, cultural beliefs, and economic factors are the main causes of treatment gaps. The overall epilepsy treatment gap in Southeast Nigeria was found to be $76 \%$, and the major contributors to the overall treatment gap were people who were never diagnosed, accounting for a diagnostic gap of $38 \%$, and those who were diagnosed but who discontinued antiepileptic drug treatment of their own volition, accounting for a therapeutic gap of $38 \%$. The epilepsy treatment gap in Southeast Nigeria is comparable to that in many other developing countries (Nwani et al., 2013).

\section{Critical determinants of the epilepsy treatment gap}

A cross-national analysis in resource-limited settings revealed that even among resourcelimited regions, people with epilepsy in countries with fewer economic, health care, neurological, and epilepsy-specific resources are more likely to have untreated epilepsy. Community-based epilepsy care programs have improved access to treatment, but in order to decrease the epilepsy treatment gap, poverty and inequalities of health care, neurology, and epilepsy resources must be dealt with at the local, national, and global levels (Meyer et al., 2012). Epilepsy is associated with substantial stigma in low-income countries, which acts as a barrier to patients accessing biomedical treatment and becoming integrated within society. Seizures can be controlled by inexpensive antiepileptic drugs, but the supply and quality of these drugs can be erratic in poor areas. The treatment gap for epilepsy is high $(>60 \%)$ in deprived areas, but this could be reduced with low-cost interventions (Newton and Garcia, 2012). The treatment gap for epilepsy is considerable in low- and middle-income countries. In the Lao PDR, the treatment gap is estimated at over $90 \%$, which might be largely contributed by the low level of knowledge of epilepsy on the part of health workers (Harimanana et al., 2013). Our investigation on treatment gaps provides an important basis to understand the distribution of epilepsy in rural areas of China and to formulate better treatment strategies as well as future prevention and control measures. 


\section{Retention rates}

Studies have only recently begun to focus on treatment retention rates. The concept of treatment based on the retention rate is intended to observe the patients using the drugs as per the treatment plan at different intervals. Thus, the proportion of subjects influenced by factors such as adverse reactions, drug efficacies, cost of treatment, and access to medication can be determined during the observation period to form a composite indicator for the long-term evaluation of the drug therapy (Aldenkamp, 2001). Studies outside of China have shown that the retention rates of the drugs lamotrigine, topiramate, ammonia acid, and gabapentin for one year were $60,55,58$, and 48\%, respectively (Marson et al., 2001). Zonisamide has also been shown to have favorable long-term retention rates, an important indication of its overall effectiveness (Brodie et al., 2012). A study of the long-term retention rate of zonisamide in a large cohort of people with epilepsy at a tertiary referral center showed that $46 \%$ of the 417 people included were still taking zonisamide at last follow-up, with an estimated 3-year retention rate of $30 \%$ (Catarino et al., 2011).

A multi-center study in epilepsy clinics in the United Kingdom evaluated the use of oral lacosamide as adjunctive therapy in adult patients with uncontrolled epilepsy. Most patients $(86.9 \%)$ presented symptomatic partial epilepsy and $80 \%$ were taking two or more antiepileptic drugs when lacosamide was introduced to the treatment (mean 2, range 0-4). Retention rates were $80 \%$ at 6 months, $68 \%$ at 1 year, and $45 \%$ at 2 years (Flores et al., 2012). Lamotrigine clearance in older adults was lower compared to younger adults in patients receiving polytherapy, polytherapy without enzyme inducers, or valproate. The 1-year retention for lamotrigine was comparable in older $(78.1 \%, 121 / 155)$ and younger $(72.4 \%, 179 / 247)$ adults in spite of the fact that younger adults tolerate lamotrigine better than older adults do. Some patients may benefit from high serum levels of lamotrigine (Arif et al., 2011). A multi-center retrospective observational study of the efficacy, tolerability, and retention rate of gabapentin use in children showed that gabapentin was well tolerated, with $55 \%$ of patients remaining on treatment beyond 1 year. The results demonstrated the efficacy and tolerability of gabapentin in children with epilepsies that are difficult to treat, and a good response to treatment beyond 12 months in both focal and generalized epilepsies (Mills et al., 2012). A study evaluating the retention rate of clobazam, topiramate, and lamotrigine in children with intractable epilepsies at 1 year demonstrated the efficacy and tolerability of the three drugs, and a good response for clobazam and lamotrigine and a reasonable response for topiramate beyond 12 months (Mills et al., 2011). The retention rate of phenobarbital in rural areas in China for 1 year was $84 \%$, and was $76 \%$ after 2 years (Wang et al., 2006). The retention rate of levetiracetam was 65 and $58 \%$ after 2 and 3 years of follow-up, respectively, although $11 \%$ of these patients had remissions within 6 months (Depondt et al., 2006). Hwang et al. (2012) suggested that ethosuximide, VPA, and lamotrigine were equally effective in the long-term treatment of newly diagnosed childhood absence epilepsy patients. Data analysis showed significantly longer retention rates with VPA versus lamotrigine treatment in overall rates as well in all syndrome subgroups. After 12 months of therapy, $69 \%$ stayed on lamotrigine therapy versus $89 \%$ on VPA, and after 24 months, these rates were 57 vs 83\%, respectively (MazurkiewiczBełdzińska et al., 2010). A comparison of the retention rates between carbamazepine and VPA as an initial monotherapy in Chinese patients with partial seizures showed that after a 10-year follow-up period, compared with VPA, patients treated with carbamazepine were more likely to discontinue treatment owing to lack of efficacy, and were less likely to discontinue antiepi- 
leptic drug treatments; however, the two differences above only occurred in the time period between the first 6 months to 2 years of treatment. Long-term treatment with carbamazepine appeared to be more effective in terms of 5-year remission and clinical control compared to VPA (Hu et al., 2011). Some researchers have compared the effectiveness of 10 antiepileptic drugs in older adults with epilepsy. The results showed that, without controlling for severity, lamotrigine had the highest 12 -month retention rate $(79 \%)$, which was significantly higher than carbamazepine (48\%), gabapentin (59\%), oxcarbazepine (24\%), phenytoin $(59 \%)$, and topiramate $(56 \%)$. The retention rate for levetiracetam $(73 \%)$ was the second highest and was significantly higher than those of carbamazepine and oxcarbazepine were (Arif et al., 2010). The EULEV cohort study evaluated the rates and factors associated with continuation of levetiracetam after 1 year. This population-based cohort study in a stable market situation found a high 1-year levetiracetam continuation rate compared with previous studies that were conducted sooner after market introduction (Droz-Perroteau et al., 2011). In our study, retention rates of $97.68,97.68,93.33$, and $43.42 \%$ were observed during the $6,12,18$, and 24 months of follow-up, respectively. Despite the significantly lower retention rate after 18 months than after 24 months, the rate was still above $40 \%$, which could still be considered a high retention rate. The treatment retention rate reflects the effectiveness and affordability of a drug for epileptic patients. Most of the subjects had generalized seizures and secondary generalized seizures. Given the effective control of their seizures using VPA, the subjects gradually discontinued the medication after the 18 months of follow-up. Thus, the retention rate after 24 months was very low. Moreover, the free supply of VPA was discontinued after the first year, which made some patients reluctant to continue with the investigation. This study was supported by the National Anti-Epilepsy Association and provided multiple advantages to the epileptic patients and their families, such as promoting the knowledge on epilepsy and helping those with financial difficulties by providing 1 year of free VPA, thereby improving the retention rate of the treatment. Reasons for withdrawal, such as economic problems, no obvious improvements of symptoms, and side effects, were noted in this study. However, being cured was still the main reason for withdrawal from the study, followed by the inconvenience of obtaining the medication. Therefore, the focus should be on health education for patients in order to inform them of the proper time to withdraw from medication.

\section{CONCLUSIONS}

This study found that the adverse reactions related to the use of VPA were mostly fatigue, drowsiness, impaired memory, abnormal liver function, dizziness, weight gain, loss of appetite, and hyperthyroidism. The total rate of adverse reactions was only $9.93 \%$, and the incidence rate was likewise low. During the 2 years of follow-up, only 8 cases discontinued the use of VPA because of these unwanted effects.

The results of our research indicated that a large treatment gap for epilepsy still exists in rural areas of China. Furthermore, VPA demonstrated high treatment efficacy with fewer side effects and higher retention rates. Therefore, antiepileptic therapy with VPA is generally effective for rural areas in China.

\section{Conflicts of interest}

The authors declare no conflict of interest. 


\section{ACKNOWLEDGMENTS}

Research supported by the National Anti-Epilepsy Association that provided multiple advantages to the epileptic patients and their families, such as promoting the knowledge on epilepsy and helping those with financial difficulties by providing 1 year of free VPA.

\section{REFERENCES}

Aldenkamp AP (2001). Cognitive and behavioural assessment in clinical trials: when should they be done? Epilepsy Res. 45: 155-157.

Arif H, Buchsbaum R, Pierro J, Whalen M, et al. (2010). Comparative effectiveness of 10 antiepileptic drugs in older adults with epilepsy. Arch. Neurol. 67: 408-415.

Arif H, Svoronos A, Resor SR Jr, Buchsbaum R, et al. (2011). The effect of age and comedication on lamotrigine clearance, tolerability, and efficacy. Epilepsia 52: 1905-1913.

Ben-Menachem E, Schmitz B, Tomson T and Vajda F (2006). Role of valproate across the ages. Treatment of epilepsy in adults. Acta Neurol. Scand. Suppl. 184: 14-27.

Brodie MJ, Ben-Menachem E, Chouette I and Giorgi L (2012). Zonisamide: its pharmacology, efficacy and safety in clinical trials. Acta Neurol. Scand. Suppl. 19-28.

Catarino CB, Bartolini E, Bell GS, Yuen AW, et al. (2011). The long-term retention of zonisamide in a large cohort of people with epilepsy at a tertiary referral centre. Epilepsy Res. 96: 39-44.

Chin JH (2012). Epilepsy treatment in sub-Saharan Africa: closing the gap. Afr. Health Sci. 12: 186-192.

Depondt C, Yuen AW, Bell GS, Mitchell T, et al. (2006). The long term retention of levetiracetam in a large cohort of patients with epilepsy. J. Neurol. Neurosurg. Psychiatry 77: 101-103.

Ding D, Lu GY, Hang MS, Zhou F, et al. (2004). Epidemiological survey on epilepsy in Shanghai rural area. Chin. J. Clin. Neurosci. 12: 122-124.

Droz-Perroteau C, Dureau-Pournin C, Vespignani H, Marchal C, et al. (2011). The EULEV cohort study: rates of and factors associated with continuation of levetiracetam after 1 year. Br. J. Clin. Pharmacol. 71: 121-127.

Flores L, Kemp S, Colbeck K, Moran N, et al. (2012). Clinical experience with oral lacosamide as adjunctive therapy in adult patients with uncontrolled epilepsy: a multicentre study in epilepsy clinics in the United Kingdom (UK). Seizure 21: 512-517.

Gekht AB (2012). Valproate (depakine chrono) in adult patients with partial epilepsy: results of a multicentral prospective non-comparative study. Zh. Nevrol. Psikhiatr. Im. S. S. Korsakova 112: 27-31.

Harimanana A, Chivorakul P, Souvong V, Preux PM, et al. (2013). Is insufficient knowledge of epilepsy the reason for low levels of healthcare in the Lao PDR? BMC Health Serv. Res. 13: 41.

Hu Y, Huang Y, Quan F, Hu Y, et al. (2011). Comparison of the retention rates between carbamazepine and valproate as an initial monotherapy in Chinese patients with partial seizures: A ten-year follow-up, observational study. Seizure 20: 208-213.

Hwang H, Kim H, Kim SH, Kim SH, et al. (2012). Long-term effectiveness of ethosuximide, valproic acid, and lamotrigine in childhood absence epilepsy. Brain Dev. 34: 344-348.

Jallon P (1997). Epilepsy in developing countries. Epilepsia 38: 1143-1151.

Karlov VA, Freìdkova NV and Rusanova LV (2012). Complex therapy of idiopathic forms of epilepsy with small doses of valproates and levetiracetam. Zh. Nevrol. Psikhiatr. Im S. S. Korsakova 112: 37-39.

Leonardi M and Ustun TB (2002). The global burden of epilepsy. Epilepsia 43 (Suppl 6): 21-25.

Marson AG, Hutton JL, Leach JP, Castillo S, et al. (2001). Levetiracetam, oxcarbazepine, remacemide and zonisamide for drug resistant localization-related epilepsy: a systematic review. Epilepsy Res. 46: 259-270.

Mazurkiewicz-Bełdzińska M, Szmuda M and Matheisel A (2010). Long-term efficacy of valproate versus lamotrigine in treatment of idiopathic generalized epilepsies in children and adolescents. Seizure 19: 195-197.

Mbuba CK, Ngugi AK, Newton CR and Carter JA (2008). The epilepsy treatment gap in developing countries: a systematic review of the magnitude, causes, and intervention strategies. Epilepsia 49: 1491-1503.

Meinardi H, Scott RA, Reis R and Sander JW (2001). The treatment gap in epilepsy: the current situation and ways forward. Epilepsia 42: 136-149.

Meyer AC, Dua T, Ma J, Saxena S, et al. (2010). Global disparities in the epilepsy treatment gap: a systematic review. Bull. World Health Organ. 88: 260-266.

Meyer AC, Dua T, Boscardin WJ, Escarce JJ, et al. (2012). Critical determinants of the epilepsy treatment gap: a cross- 
national analysis in resource-limited settings. Epilepsia 53: 2178-2185.

Mills JK, Lewis TG, Mughal K, Ali I, et al. (2011). Retention rate of clobazam, topiramate and lamotrigine in children with intractable epilepsies at 1 year. Seizure 20: 402-405.

Mills JK, Ruslan NE, Lewis TG, Mughal K, et al. (2012). Retention rate of Gabapentin in children with intractable epilepsies at 1 year. Seizure 21: 28-31.

Newton CR and Garcia HH (2012). Epilepsy in poor regions of the world. Lancet 380: 1193-1201.

Nwani PO, Nwosu MC, Enwereji KO, Asomugha AL, et al. (2013). Epilepsy treatment gap: prevalence and associated factors in Southeast Nigeria. Acta Neurol. Scand. 128: 83-90.

Ratsimbazafy V, Andrianabelina R, Randrianarisona S, Preux PM, et al. (2011). Treatment gap for people living with epilepsy in Madagascar. Trop. Doct. 41: 38-39.

Scott RA, Lhatoo SD and Sander JW (2001). The treatment of epilepsy in developing countries: where do we go from here? Bull. World Health Organ. 79: 344-351.

Sridharan R and Murthy BN (1999). Prevalence and pattern of epilepsy in India. Epilepsia 40: 631-636.

Wang DS, Li GZ, Ma GY and Tang Y (2002a). Epidemiological survey on epilepsy in Dongning County, Hei Long Jiang province in rural. Chin. J. Stroke Neurol. 19: 159-161.

Wang WZ, Wu JG, Wang DS, Chen G, et al. (2002b). Sample survey about the epilepsy epidemic of population in rural areas of five province in China. J. Chin. Med. 82: 449-452.

Wang WZ, Wu JZ, Wang DS, Dai XY, et al. (2003). The prevalence and treatment gap in epilepsy in China: an ILAE/IBE/ WHO study. Neurology 60: 1544-1545.

Wang WZ, Wu JZ, Ma GY, Dai XY, et al. (2006). Efficacy assessment of phenobarbital in epilepsy: a large communitybased intervention trial in rural China. Lancet Neurol. 5: 46-52.

Wang WZ, Wu JZ, Li SC, Ruo XJ, et al. (2011). Effect evaluation of the medical treatment of epilepsy by VPA for 607 cases in rural areas in China. Chin. J. Neurol. 44: 15-18.

Wang WZ, Wu JZ, Li SC, Ru XJ, et al. (2012). Sodium valproate for epilepsy in rural China: an efficacy and safety assessment in primary care. Epilepsy Res. 102: 201-205.

Wu X (2006). Standard treatment is the key to improve epilepsy. Chin. J. Internal Med. 45: 798-799.

Zhao YH, Zhou D, Zhang Q, Zhaxi CR, et al. (2007). Tibet preliminary investigation on the prevalence of seizures in patients with epilepsy in rural areas. Chin. J. Neurol. 40: 580-583. 